\title{
Approximate Soliton Solutions of Real Order Sine- Gordon Equations
}

\author{
Neslihan ÜZAR \\ Istanbul University, Science Faculty, Department of Physics, Vezneciler, 34134 Istanbul, Turkey. \\ e-posta: neslihanuzar@gmail.com
}

Geliş Tarihi: 28.05.2016 ; Kabul Tarihi: 22.05.2017

$\begin{array}{cl}\text { Keywords } & \text { attained remarkable result from these solutions. While the solutions of classical and time-fractional SG } \\ \text { Caputo fractional } & \begin{array}{l}\text { equations are kink of type (Although of being the same type, they are different from each other), } \\ \text { solution of the space-fractional SG equation is breather of type i.e., different types of soliton solutions } \\ \text { derivative operator; } \\ \text { HPM; SG equation; }\end{array} \begin{array}{l}\text { are obtained using similar initial conditions for time and space fractional SG equation. Also these results } \\ \text { fractional non-linear } \\ \text { equation. }\end{array} \quad \begin{array}{l}\text { dispersion of fiber optics communication can be modelled by fractional SG equations. In other words, } \\ \text { this study may be very important for bringing to light the real behaviour of physical systems which have } \\ \text { usually been described by classical SG equation. Because some physical events such as the memory } \\ \text { effects of non-Markovian processes, the effects of non-Gaussian distribution, interactions between the } \\ \text { systems and environment and some physical losses in the systems which are neglected in classical SG } \\ \text { equation can be taken into account with fractional SG equations. }\end{array} \\ \end{array}$

\section{Reel Mertebeli Sine-Gordon Denklemlerinin Yaklaşık Soliton Çözümleri}

\section{Anahtar kelimeler \\ Caputo kesirsel türev operatörü; HPM; SG denklemi; kesirli lineer olmayan denklemler}

\begin{abstract}
Özet
Bu çalışmada kesirli Sine-Gordon denklemleri (zaman-kesirli, uzay-kesirli ve zaman-uzay-kesirli) homotopy pertürbasyon metodu (HPM) kullanılarak çözülmüştür. Bu çözümlerden dikkat çekici sonuçlar elde edilmiştir. Klasik ve zaman-kesirli SG denklemlerinin çözümü kink tipi iken (aynı tipte olmalarına rağmen, birbirlerinden farklıdır), uzay-kesirli SG denkleminin çözümü breather tiptir; başka bir ifadeyle zaman ve uzay kesirli SG denklemi için benzer başlangıç koşulları kullanıldığında farklı tip soliton çözümleri elde edilmiştir. Ayrıca bu sonuçlar josephson eklemlerindeki vorteks-antivorteks çifleri veya fiber optik iletişimde sinyal dağılımındaki kayıplar gibi bazı olayları kesirli SG denklemleri ile modelleyebileceğini göstermiştir. Diğer bir deyişle, bu çalışma genellikle klasik SG denklemleri ile tanımlanan fiziksel sistemlerin gerçek davranışlarına ışık tutabilir. Çünkü Markovian olmayan süreçlerin bellek etkileri, Gaussian olmayan dağılımların etkileri, sistem ile dış çevre arasındaki etkileşmeler ve klasik SG denkleminin ihmal ettiği sistemler içerisindeki bazı fiziksel kayıplar gibi bazı fiziksel olaylar kesirli SG denklemleri ile hesaba katılabilir.
\end{abstract}

(C) Afyon Kocatepe Üniversitesi

\section{Introduction}

The classical SG equation being a nonlinear partial differential equation has firstly been created by Edmond Bourin during the study of negative but constant Gaussian curvature $K=-1$ surface in
1862 (Bracken 2011). Later in 1939 the dislocation of similar atoms in a chain was defined using this equation (Frenkel et al. 1939). Its importance greatly increased when kink and anti-kink solutions possessing the collisional properties of 
solitons were found (Int sour. 1). The classical SG equation is also used in a number of other physical applications. Among these are the motion of a rigid pendulum attached to a stretched wire Jin (2009); Wazwaz (2012), the dynamical properties of DNA molecules Wazwaz (2012), the stimulation of phonon modes Aktosun et al. (2010), the signal dispersion in fiber optics for long-distance communications (Int sour. 2) and the phase difference across a long Josephson junction carrying a current infinitely under any applied voltage through two superconductors separated by an insulator layer Derks et al. (2003). The classical SG equation in normalized units is Kaya (2003); Batiha et al. (2007)

$$
\frac{\partial^{2} u}{\partial t^{2}}-\frac{\partial^{2} u}{\partial x^{2}}+\sin u=0
$$

for $u \equiv u(x, t)$.

The classical SG equation description of behaviours occurring in physical systems is considered to be of superficial form; because this equation is somehow an idealization and therefore cannot bear in itself some processes occurring in nature, due to the complexity of real phenomena. This is so, since all quantum systems always interact with their surroundings and hence are open. Also, strong interactions between particles in the systems, the effects of the fractallity (fractal nature of space where occurrence of physical events are realized) and memory effects of non-Markovian processes lead to dissipation and quantum decoherence due to the transition of information, energy and particles flows. All of these effects cause irreversibility, non-conservation, in-homogeneousness and nonunitarity in systems.

Considering these facts, many scientists, who are in different fields such as physics, mathematics, economy..., have been started to work out on fractional linear and nonlinear differential equations. Especially, in physics fractional equations with fractional derivative and integral operators have been used for explaining the cases where the laws of classical physics are inadequate (Tsallis 1988; Tarasov 2005; Metzler et al. 2000) and some physical events such as memory effects can be better described with fractional operator
(Akgul et al. 2015). Therefore, in this study we intend to consider the generalized SG equations with fractional differo-integral operators and to numerically solve them using HPM, carrying the hope that we can find solutions with some interesting properties corresponding to real behaviours. Also this study has attempted to shed light on the important issue of solid state physics such as optical fiber communication and Josephson junction (Ray 2015). These two physical systems have got soliton behaviour and all losses in these systems can be explained with fractional SG equation. There are a few of studies about numerical solution of fractional Sine -Goedon equation with using different method (Akgul et al. 2016; Yousef et al. 2016; Pandir 2016) and to our knowledge there isn't any study about spacetime- fractional SG equation in the literature. Here different initial conditions, which were not used previously in the literature, were used for solving these SG equations. Also, since we are going to consider here the fractional SG equations we have to use different definition of sine function which is created by fractional numbers. We think that this paper would make an important contribution to the literature because of these new approximations and interesting results. Some general information is given for using in the solution phase as follows.

\section{Materials and Methods}

\subsection{Basic definitions for fractional calculus}

There are several derivative and integral operator definitions which are used for fractional calculus such as Riemann-Liouville, Caputo and GrünwaldLetnikov (Podlubny 1999). Caputo's definition is the most successful one in expressing the real world phenomena (Ateş 2010). Also it reduces to the ordinary derivative operator for integer-order values of the fractional parameter and allows us to take the initial and boundary conditions in the same form used in integer order differential equations (Podlubny 1999). The temporal Caputo fractional derivative operator is expressed in the following form (Podlubny 1999; Miller et al. 1993; Oldham et al. 1974; Caputo 1967). 
$D^{\beta} f(t)=\left\{\begin{array}{lll}J^{n-\beta} \frac{\mathrm{d}^{n}}{\mathrm{~d} t^{n}} f(\tau) & \text { for } & n-1<\beta \leq n \\ \frac{\mathrm{d}^{n}}{\mathrm{~d} t^{n}} f(t) & \text { for } & n=\beta\end{array}\right.$

for $n \in N$, where $J^{n-\beta}$ is the Riemann-Liouville fractional integral operator defined as, for $\beta>0$ and $t>0$

$$
J^{\beta} f(t)=\frac{1}{\Gamma(\beta)} \int_{0}^{t}(t-\tau)^{\beta-1} f(\tau) d \tau .
$$

We shall also utilize the following equations during our calculations (Podlubny 1999; Miller et al. 1993; Oldham et al. 1974; Caputo 1967).

$$
\begin{aligned}
& D^{\beta} J^{\beta} f(x)=f(x) \text { and } \\
& J^{\beta} D^{\beta} f(x)=f(x)-\sum_{k=0}^{n-1} f^{(k)}\left(0^{+}\right) \frac{x^{k}}{k !}
\end{aligned}
$$

\subsection{Fractional SG equations}

Below we present time-fractional, space-fractional and time-space-fractional SG equations, written using Caputo definition, respectively

$$
\begin{array}{ll}
{ }_{0} D_{t}^{\alpha} u-\frac{\partial^{2} u}{\partial x^{2}}+\sin u=0, & 0<\alpha \leq 2 \\
\frac{\partial^{2} u}{\partial t^{2}}-D_{x}^{\beta} u+\sin u=0, & 0<\beta \leq 2 \\
{ }_{0} D_{t}^{\alpha} u-D_{x}^{\beta} u+\sin u=0, & 0<\alpha \leq 2,0<\beta \leq 2 .
\end{array}
$$

The memory effects, the strong interactions between particles and some restrictions about physical systems can be taken into account with the time-fractional SG equation; whereas the fractallity of the space can be taken into account with the space-fractional SG equation and all of the above mentioned phenomena can clearly be taken into account with the time-space-fractional SG equation.

\subsection{Homotopy perturbation method}

HPM is a powerful method for solving all of classical or fractional ordinary and partial differential equations easily (He 2000; El-Shahed 2005; He 2004; He 2005; Momani et al. 2007). Actually HPM gives only an approximate solution, not an exact one. In this method, in solving the target differential equation, a guess initial solution is suggested and the final solution is obtained using this guess initial solution with a number of perturbative operations. Our fractional nonlinear partial differential equations will be solved with this method; therefore basic information about how to solve a nonlinear equation with HPM is given here. Firstly consider the following differential equation

$$
A(\phi)-f(x, t)=0
$$

which satisfies the boundary condition

$$
B\left(\phi(x, t), \frac{\partial \phi(x, t)}{\partial t}\right)=0 .
$$

Where $A(\phi)$ is a non-linear partial differential equation, $\phi(x, t)$ is the unknown function depending on the independent variables $x$ and $t . B$ is the boundary operator and $f(x, t)$ is a known constant or function $A(\phi)$ can be divided into two parts which include the linear $L(\phi)$ and nonlinear $N(\phi)$ terms. Therefore Eq. (3) can be written as

$$
L(\phi)+N(\phi)-f(x, t)=0 .
$$

According to the definitions of HPM, a homotopy function is constructed as $\phi((r, t), p): \Omega \times[0,1] \rightarrow \mathrm{R} \quad$ satisfying the following equations.

$$
\begin{aligned}
H(\phi, p) & =(1-p)\left[L(\phi)-L\left(\psi_{0}\right)\right]+p[A(\phi)-f(x, t)] \\
& =L(\phi)-L\left(\psi_{0}\right)+p L\left(\psi_{0}\right)+p[N(\phi)-f(x, t)]=0
\end{aligned}
$$

where $p$ is a parameter changing from 0 to $1 . \psi_{0}$ is the initial guess solution of Eq.(3) and $\phi_{0}=\psi_{0}$ is generally accepted. In general, the approximate solution function depends on time and space (only $x$ as considered in this paper). The solution of the differential equation changes from $\phi_{0}=\psi_{0}$ to $\phi(x, t)$ when $p$ changes from 0 to 1 . Additionally, this initial guess solution must satisfy the boundary conditions of Eq. (3). 
The final solution can be written as a Taylor series according to the powers of $p$ :

$$
\phi=\phi_{0}+p \phi_{1}+p^{2} \phi_{2}+\ldots=\sum_{i=0}^{\infty} p^{i} \phi_{i}
$$

This expression is substituted in Eq. (4) and the solution functions $\phi_{i}$ are found according to the powers of $p$. Then substituting 1 for $p$, the approximate solution is obtained.

\section{The Numerical Solutions of Fractional SG Equations}

There are a few studies on time fractional and space fractional SG equations. Only $u-\frac{u^{g}}{3 !}$ terms in the Taylor series of sine function which is created by integer number $\left(\sin u=\sum_{n=1}^{\infty} \frac{(-1)^{n-1} u^{2 n-1}}{(2 n-1) !}\right)$ are taken to solve time fractional SG equation with Caputo fractional derivative operator for different initial conditions (Elsaid et al. 2012). At this study and other studies Lu (2009); Jin (2009) consider the Taylor series expansion of $\sin (u)$ to obtain the approximate solution. The other studies have been done on the space fractional SG equation with Riesz fractional derivative operator using modified decomposition method with Fourier transform Int sour.4; Miskinis (2005); Saha Ray (2016) or using fractional EulerLagrange equations (Nasrolahpour 2013) and reproducing kernel method (Akgul 2016). Almost similar results were obtained in most of these studies. To our knowledge there isn't any study about space-time- fractional SG equation in the literature and here different initial conditions, which were not used previously in the literature, were used for solving these SG equations. Also, since we are going to consider here the fractional SG equations we have to use the following definition of sine function which is created by fractional numbers in Eq. (6).

$$
\sin u=i \frac{u^{2}}{2 !}-i \frac{u^{4}}{4 !}+i \frac{u^{6}}{6 !}-\cdots+\frac{(-1)^{\frac{n}{2}-1} u^{2 \frac{n}{2}-1}}{\left(2 \frac{n}{2}-1\right) !} \cdots
$$

\subsection{Time-fractional SG Equation}

Contrary to the above mentioned Ref Elsaid et al. (2012) we are going to consider the term of Taylor series of sine function up to $14^{\text {th }}$ degree. The solution of time fractional was obtained for 2, 4, 6, $8,10,12$ and $14^{\text {th }}$ terms of Taylor series of sine function, respectively. Under the approximation which is given in Eq. (6), the time fractional SG equation becomes

$$
{ }_{0} D_{t}^{\alpha} u-\frac{\partial^{2} u}{\partial x^{2}}+i \frac{u^{2}}{2 !}=0
$$

$$
{ }_{0} D_{t}^{\alpha} u \frac{\partial^{2} u}{\partial x^{2}}+i \frac{u^{2}}{2 !}-i \frac{u^{4}}{4 !}+i \frac{u^{6}}{6 !}-i \frac{u^{8}}{8 !}+i \frac{u^{10}}{10 !}-i \frac{u^{12}}{12 !}+i \frac{u^{14}}{14 !}=0
$$

Its solution obtained by second order HPM, with $\psi_{0}=u_{0}=u(x, 0)=4 \arctan \mathrm{e}^{\frac{x}{k}}$. Here not all of the numerical and graphical results for the above equations are given. Only approximate solution is given for $4^{\text {th }}$ terms of sine function as following.

$$
\begin{aligned}
& u(x, t)=4 \arctan \mathrm{e}^{\frac{x}{k}}-\frac{1}{9\left(1+\mathrm{e}^{\frac{2 x}{k}}\right)^{4} k^{4} \Gamma\left[\frac{1}{2}+\alpha\right] \Gamma[1+\alpha]} \times \\
& \times 4^{1-\alpha} \sqrt{\pi} t^{2 \alpha}\left(9 \mathrm{e}^{\frac{x}{k}}\left(-1+23 \mathrm{e}^{\frac{2 x}{k}}-23 \mathrm{e}^{\frac{4 x}{k}}+\mathrm{e}^{\frac{6 x}{k}}+4 i \mathrm{e}^{\frac{x}{k}} k^{2}+8 \mathrm{e}^{\frac{3 x}{k}} k^{2}+4 \mathrm{e}^{\frac{5 x}{k}} k^{2}\right)\right. \\
& -72 \mathrm{e}^{\frac{x}{k}}\left(-1+\mathrm{e}^{\frac{2 x}{k}}\right)\left(1+\mathrm{e}^{2 \frac{2 x}{k}}\right) k^{2} \arctan \mathrm{e}^{\frac{x}{k}}-288 \mathrm{i}^{\frac{2 x}{k}}\left(1+\mathrm{e}^{2 \frac{2 x}{k}}\right) k^{2} \arctan \mathrm{e}^{\frac{2 x}{k}} \\
& +24\left(1+\mathrm{e}^{\frac{2 x}{k}}\right)^{2} k^{2}\left(-8 i \mathrm{e}^{\frac{x}{k}}+8 \mathrm{e}^{\frac{3 x}{k}}+3 k^{2}+6 \mathrm{e}^{\frac{2 x}{k}} k^{2}+3 \mathrm{e}^{\frac{4 x}{k}} k^{2}\right) \arctan \mathrm{e}^{\frac{3 x}{k}}
\end{aligned}
$$




$$
\left.-288\left(1+\mathrm{e}^{4 \frac{2 x}{k}}\right) k^{4} \arctan \mathrm{e}^{5 \frac{x}{k}}+256\left(1+\mathrm{e}^{4 \frac{2 x}{k}}\right) k^{4} \arctan \mathrm{e}^{7 \frac{x}{k}}\right)
$$$$
+\frac{8 i \mathrm{e}^{\frac{2 x}{k}} t^{\alpha}\left(4 k^{2} \arctan \mathrm{e}^{2 \frac{x}{k}}\left(-3+4 \arctan \mathrm{e}^{2 \frac{x}{k}}\right) \cosh \frac{x^{2}}{k^{2}}-3 i \sinh \frac{x}{k}\right)}{3\left(1+\mathrm{e}^{\frac{2 x}{k}}\right)^{2} k^{2} \Gamma[1+\alpha]}
$$

$\Gamma$ is the gamma function. $u(x, t)$ is evidently complex.

The probability density graphs belonging to the above kink of type solution are given in Fig.1 versus $x$ and $t$ for different values of $\alpha$ and $k=0.8$. The graphs in Fig. 1 break down at the same position as time progresses. So it can be thought that the system is non-conserved, irreversible and inhomogeneous and possess nonGaussian distributions for non-integer $\alpha$ values. However as $\alpha$ approaches 2 the situation changes and a Gaussian probability density appears again. (a)

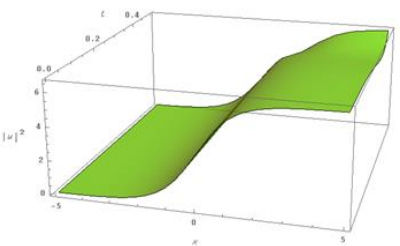

(c)

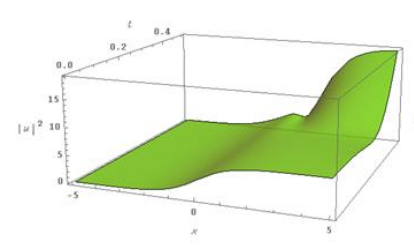

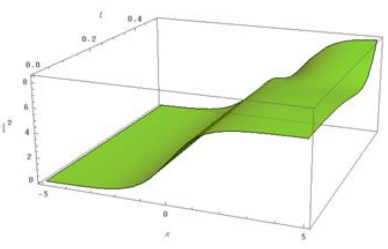

(d)

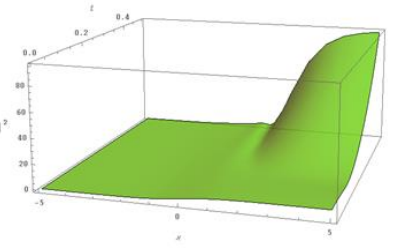

Fig. 1. The probability density of solution of timefractional SG equation for $4^{\text {th }}$ term of sine function with initial condition $\phi_{0}=u_{0}=4 \arctan \mathrm{e}^{\frac{x}{k}}$ for (a) $\alpha=1.9$, (b) $\alpha=1.8$, (c) $\alpha=1.6$ and (d) $\alpha=1.2$.

Kink of type solution is obtained for Eqs. (7a-7g). As the number of the Taylor expansion terms lowers distortions in the figure become more and more and as the number of the terms increases the difference between the solutions becomes less and finally almost vanishes (Fig. 2). (a)

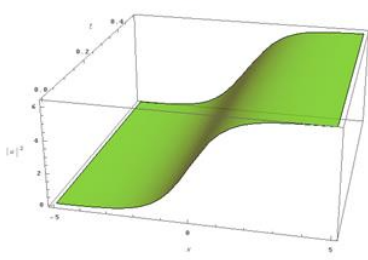

(c)

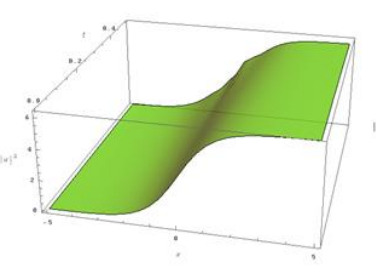

Fig. 2. The probability density of solution for (a) $\alpha=1.9$ with $14^{\text {th }}$ terms (b) $\alpha=1.9$ with $10^{\text {th }}$ terms (c) $\alpha=1.2$ with $14^{\text {th }}$ terms (d) $\alpha=1.2$ with $10^{\text {th }}$ terms depending on $x$ and $t$.

Time fractional SG equation approaches the classical SG equation and, it was observed that the $\alpha$ dependence of solution is almost eliminated for higher terms. The solution moves away from the classical one for the lowest valued parameter $\alpha=1.2$ for all terms.

For comparison we have to consider the solution of classical SG equation and the graph of mod square of $u(x, t)$ versus $x$ and $t$. Under the approximation about sine function with Taylor series expanded, the classical SG equation takes the form

$\frac{\partial^{2} u}{\partial t^{2}}-\frac{\partial^{2} u}{\partial x^{2}}+u-\frac{u^{3}}{3 !}=0$

The solution is going to be obtained with second order HPM using the initial guess solution as $\psi_{0}=u_{0}=u(x, 0)=\arctan \mathrm{e}^{\frac{x}{k}}$ which comes from the kink soliton solution of classical SG equation by taking the initial position $x_{0}=0$ and $k=\sqrt{1-c^{2}}$, with $c$ the propagation of soliton (Int sour. 1) The approximate solution is 
$u(x, t)=\arctan \mathrm{e}^{\frac{x}{k}}+\frac{\mathrm{e}^{4 \frac{x}{k}} t^{4}}{36 k^{4}\left(1+\mathrm{e}^{2 \frac{x}{k}}\right)^{4}} \times$

$\times\left[\left(69+6 k^{2}\right) \sinh \frac{x}{k}+k^{2} \arctan \mathrm{e}^{\frac{x}{k}} \cosh \frac{x^{2}}{k^{2}}\left(6+12 k^{2}+k^{2} \arctan \mathrm{e}^{2 \frac{x}{k}}\left(-8+\arctan \mathrm{e}^{2 \frac{x}{k}}\right.\right.\right.$

$\left.\left.+k^{2}\left(12-8 \arctan \mathrm{e}^{2 \frac{x}{k}}+\arctan \mathrm{e}^{4 \frac{x}{k}}\right) \cosh \frac{2 x}{k}-12 \arctan \mathrm{e}^{\frac{x}{k}} \sinh \frac{x}{k}\right)+3\left(-1+2 k^{2}\right) \sinh \frac{3 x}{k}\right] \mathrm{c}$

$+\frac{t^{2}}{12 k^{2}}\left[k^{2} \arctan \mathrm{e}^{\frac{x}{k}}\left(-6+\arctan \mathrm{e}^{2 \frac{x}{k}}\right)-3 \operatorname{sech} \frac{x}{k} \tanh \frac{x}{k}\right]$.

These extra terms are due to the consideration of the last term. The probability density graph belonging to this solution is shown in Fig. 3 for $k=0.8$, depending on both $x$ and $t$.

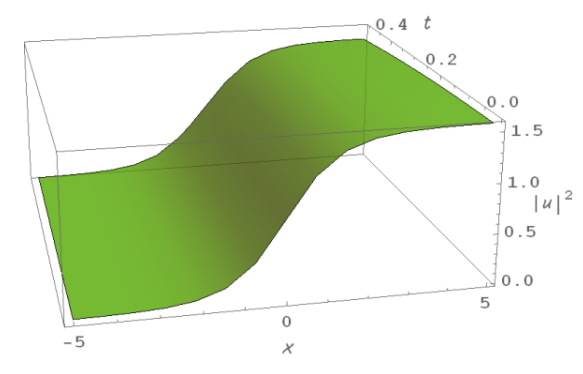

Fig. 3. Graph of probability density of classical SG equation solution depending on $x$ and $t$.

The most striking aspect of nonlinear differential equations is that they have solutions which show different behaviours under different initial conditions and parameter values. Here kink of type solution is obtained depending on the chosen initial guess solution. It does not seem a disorder at the graph in Fig. 3 as time progresses. In other words, when the time changes, there is no variation of probability density for the same position. It may suggest that this system is conserved, reversible and homogenous and is expected since all losses in the system and interactions with environment have been neglected. The system described by the integer derivatives is local in the vicinity of any point in space and time, i.e., the system is described in a rough draft way. Also we have done the calculations up to $15^{\text {th }}$ power and see no difference between the two results.

Fig.4a and $4 \mathrm{~b}$ depict the probability density function against the fractional order

parameter $\alpha$ for Eq.(7b) and Eq.(7g), respectively. $t=0.5$ and $x=1$ are assumed for the numerical representations. A nearly exponential behaviour is observed from Fig. 4a. Here the probability of occurrence of physical events is higher always and $\frac{x}{k}$ decreases as $\alpha$ increases and tends almost to zero in the vicinity of $\alpha=2$. Fig. $4 \mathrm{~b}$ belongs to the $14^{\text {th }}$ order solution and shows clearly that $\alpha$ dependence of solution disappears at higher orders.

(a)

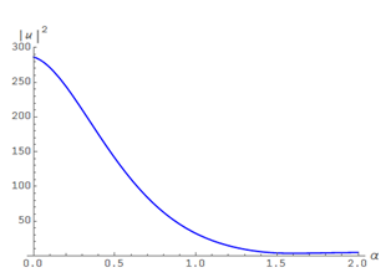

Fig. 4. The graphical representation of variation of $|u|^{2}$ against $\alpha$ for (a) $4^{\text {th }}$ terms, (b) $14^{\text {th }}$ terms of Taylor series of sine function.

In fact, the real systems are always under some interactions, restrictions and enforcement due to the flow of information and energy with their environment. These cause such systems to be excited, complex and random. Moreover for realistic systems there are flows of information from the environment back to the system. That is the earlier states of any system affect its future states; in other words, the future and past of the system are interdependent. This is the realization of memory effects. Hence such systems, open systems can be defined only with stochastic (probability) processes. On the other hand classical SG equation does not include memory effects since it describes the system at a specific time $t$. This means that many physical phenomena are neglected and the system is in a steady state. Any open system only reaches a steady state when all losses of information and energy come to an end. Combining the obtained results we conclude that time-fractional SG equation is suitable for representing the memory effects of non-Markovian processes.

\subsection{Space-fractional SG Equation}

The effects of space fractallity arising from the non-uniformity of particles density and scattering 
between particles shows up itself in Eq.(2b). The space fractional SG equation was solved for 2, 4, $6,8,10,12$ and $14^{\text {th }}$ terms of Taylor series of sine function, respectively in this section. Under the approximation that we have followed up to, this equation takes the form

$$
\frac{\partial^{2} u}{\partial t^{2}}-D_{x}^{\beta} u+i \frac{u^{2}}{2 !}-i \frac{u^{4}}{4 !}+i \frac{u^{6}}{6 !}-\cdots+i \frac{u^{14}}{14 !}=0 \quad 0<\beta \leq 2 .
$$

The second order HPM solution of these equations was obtained starting with the initial condition $\psi_{0}=u_{0}=u(0, t)=4 \arctan \mathrm{e}^{\frac{t}{k}}$. Here only the numerical and graphical solutions of $4^{\text {th }}$ terms of sine function are given. The numerical result is found as

$$
u(x, t)=4 \arctan \mathrm{e}^{\frac{t}{k}}-\frac{1}{9\left(1+\mathrm{e}^{\frac{2 t}{k}}\right)^{4} k^{4} \Gamma\left[\frac{1}{2}+\beta\right] \Gamma[1+\beta]} \times
$$

$\times 4^{1-\beta} \sqrt{\pi} x^{2 \beta}\left(9 \mathrm{e}^{\frac{t}{k}}\left(-1+23 \mathrm{e}^{\frac{2 t}{k}}-23 \mathrm{e}^{\frac{4 t}{k}}+\mathrm{e}^{\frac{6 t}{k}}-4 i \mathrm{e}^{\frac{1}{k}} k^{2}-8 \mathrm{i}^{\frac{3 t}{k}} k^{2}-4 i \mathrm{e}^{\frac{5 t}{k}} k^{2}\right)+72 i \mathrm{e}^{\frac{1}{k}}\left(-1+\mathrm{e}^{\frac{2 t}{k}}\right)\left(1+\mathrm{e}^{\frac{2 t}{k}}\right)^{2} k^{k}\right.$ $+288 \mathrm{e}^{\frac{2 t}{k}}\left(1+\mathrm{e}^{\frac{2 t}{k}}\right)^{2} k^{2} \arctan \mathrm{e}^{2 \frac{t}{k}}+24\left(1+\mathrm{e}^{\frac{2 t}{k}}\right)^{2} k^{2}\left(8 \mathrm{e}^{\frac{t}{k}}-8 i \mathrm{e}^{\frac{3 t}{k}}+3 k^{2}+6 \mathrm{e}^{\frac{2 t}{k}} k^{2}+3 \mathrm{e}^{\frac{4 t}{k}} k^{2}\right)$ $\left.-288\left(1+\mathrm{e}^{\frac{2 t}{k}}\right)^{4} k^{4} \arctan \mathrm{e}^{5 \frac{t}{k}}+256\left(1+\mathrm{e}^{\frac{2 t}{k}}\right)^{4} k^{4} \arctan \mathrm{e}^{7 \frac{t}{k}}\right)$

$$
-\frac{8 i \mathrm{e}^{\frac{2 t}{k}} x^{\beta}\left(4 k^{2} \arctan \mathrm{e}^{2 \frac{t}{k}}\left(-3+4 \arctan \mathrm{e}^{2 \frac{t}{k}}\right) \cosh \frac{t^{2}}{k^{2}}+3 i \sinh \frac{t}{k}\right)}{3\left(1+\mathrm{e}^{\frac{2 t}{k}}\right)^{2} k^{2} \Gamma[1+\beta]}
$$

The fractional parameter $\beta$ which is of the order of space derivative operator is the same as the scale of fractal structure of space. It plays a fundamental role on the probability density versus $x$ and $t$, for $k=0.8$ (Fig.5). These graphs which are not complying with central limit theorem show that space fractional SG equation has nonconserved and non-Gaussian distribution. However as $\beta$ approaches 2 , the system receives a nearly Gaussian distribution.

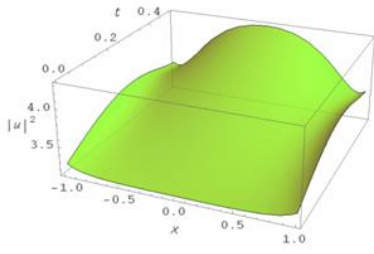

(c)

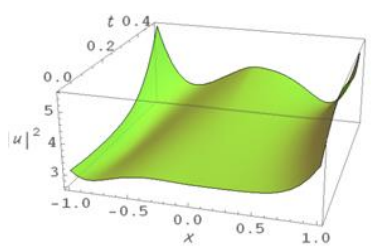

(b)

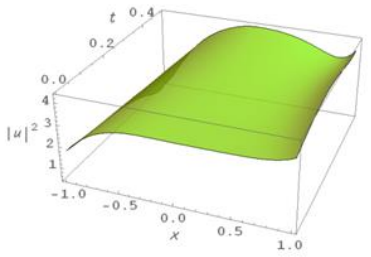

(d)

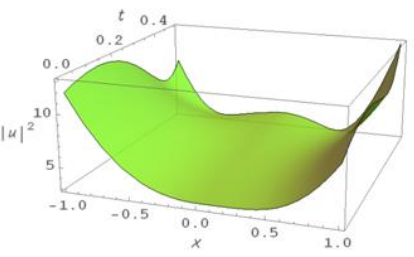

Fig. 5. The graphs of probability density of $u(x, t)$ with $4^{\text {th }}$ terms of sine function for (a) $\beta=1.9$, (b) $\beta=1.8, \quad$ (c) $\beta=1.6$ and (d) $\beta=1.2$

The graphical results for different terms of Taylor series of sine function show that solutions are nearly same. But there are some differences. Especially, the result is the same at $\beta=1.2$ for all orders. All numerical and graphical results are not given in this paper because of they occupy a lot of space. To compare between the obtained solutions, some graphical results are given with fractional parameter $\beta=1.2$ and 1.9 for $10^{\text {th }}$ and $14^{\text {th }}$ terms of sine function.

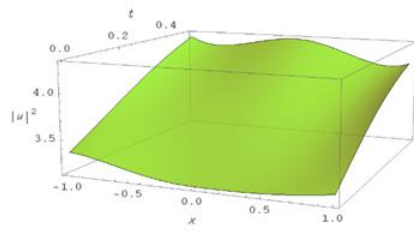

(c)

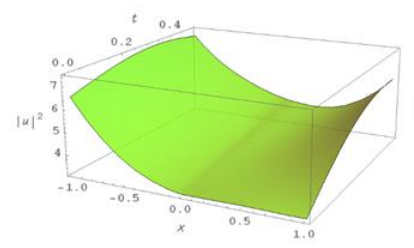

(b)

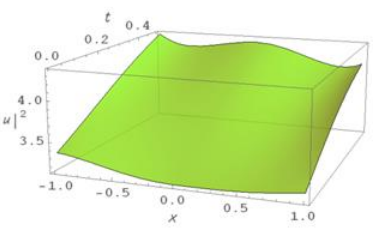

(d)

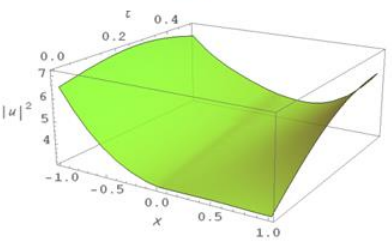

Fig. 6. The probability density of space- fractional SG equation solution for (a) $\beta=1.9$ with $14^{\text {th }}$ terms (b) $\beta=1.9$ with $10^{\text {th }}$ terms $\quad$ (c) $\beta=1.2$ with $14^{\text {th }}$ terms (d) $\beta=1.2$ with $10^{\text {th }}$ terms depending on $x$ and $t$.

These obtained results are also supported by Fig.7a and $7 \mathrm{~b}$ which depict the graphical 
representation of probability density function versus the space- fractional parameter $\beta$ for $4^{\text {th }}$ terms and $10^{\text {th }}$ terms of Taylor series, respectively. Here $x=1$ and $t=0.5$. The $\beta$ dependence of mod square of $u$ is the same graphically for all orders, but it varies as numerical value.

(a)

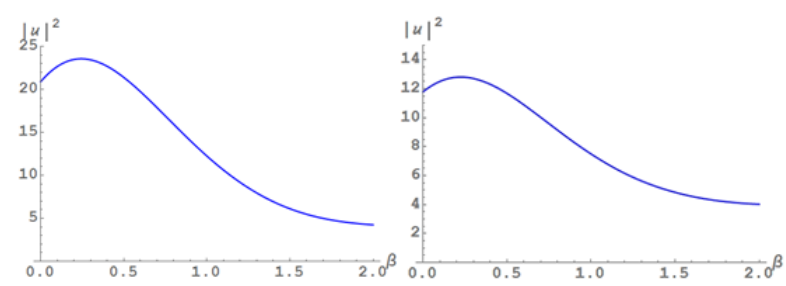

Fig. 7. Graphical representation of probability of solution functions versus the space- fractional parameter $\beta$ for (a) $4^{\text {th }}$ terms, (b) $10^{\text {th }}$ terms . Here $x=1$ and $t=0.5$ are taken.

The crucial point is the attained remarkable result. While the solution of classical and time-fractional SG equation was kink of type, it is clearly seen from Figs. 5 and 6 that this time the solution is breather of type. Although kink of type soliton is a particle of type solution; breather of type soliton is a non-linear wave. Therefore kink and breather of type solutions are known as topological and non-topological solitons, respectively. While kink of type solution is non-localized, breather of type soliton is localized periodic solution. Actually, breather of type soliton corresponds to a collision of two different of type solitons; the kink-antikink solitons (Int sour. 3). By using this feature, some losses in physical systems can be explained; such as vortex-antivortex couples in a Josephson junction or scattering of signal flux and antiflux in a fiber optics system (Ivancevic Vladimir et al. 2013). For these reasons this result may be important to explain some physical events.

\subsection{Time and Space-Fractional SG Equation}

Finally, let us consider Eq. (2c) under the approximation with $2,4,6,8,10,12$ and $14^{\text {th }}$ terms of Taylor series of sine function, respectively.

$$
{ }_{0} D_{t}^{\alpha} u-D_{x}^{\beta} u+i \frac{u^{2}}{2 !}=0,0<\alpha \leq 2,0<\beta \leq 2
$$

$$
{ }_{0} D_{t}^{\alpha} u-D_{x}^{\beta} u+i \frac{u^{2}}{2 !}+\cdots+i \frac{u^{14}}{14 !}=0,0<\alpha \leq 2,0<\beta \leq 2
$$

Only the first order HPM solution is taken for initial condition $\psi_{0}=u_{0}=u(x, 0)=4 \arctan \mathrm{e}^{\frac{x}{k}}$ in this case, because of the complex structure of the equation. The obtained approximate solution is given for the $4^{\text {th }}$ terms of power series of sine function as following.

$u(x, t)=4 \arctan \mathrm{e}^{\frac{x}{k}}+\frac{8 i t^{\alpha} \arctan \mathrm{e}^{2 \frac{x}{k}}\left(-3+4 \arctan \mathrm{e}^{2 \frac{x}{k}}\right)}{3 \Gamma[1+\alpha]}$

$$
-\frac{2\left(t^{\alpha}-(t-x)^{\alpha}\right) x^{2-\beta} \operatorname{sech} \frac{x}{k} \tanh \frac{x}{k}}{k^{2} \Gamma[1+\alpha] \Gamma[3-\beta]}
$$

This example describes a real system with considering both non-Markovian and nonGaussian distribution effects. For a specific time value chosen as 0.1 , the plot of probability density of the above solution is shown in Fig. 8 versus $x$. Here one clearly sees the non-Markovian and nonGaussian effects together since the curve represents both breather and kink of type solitons. Here $k=0.8$ is accepted for the graphical representations.

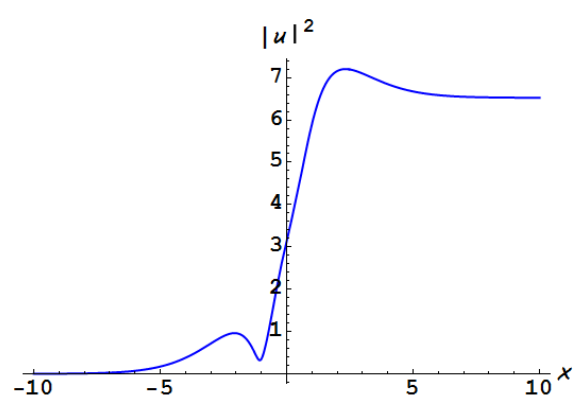

Fig. 8. The graph of probability density of time and space -fractional SG equation versus $x$ at $t=0.1$. Both of the breather and kink of type soliton characteristics are seen in the graph, clearly. 
The difference in the space-time fractional SG equation for same parameter in all terms is very little. We can say that the order of power series of sine function does not affect the graphical solution. When the second-order HPM solutions carried out, the effect of the order of power series of sine function is not observed again. Only second order HPM solution gives the more detail result (Fig. 9).

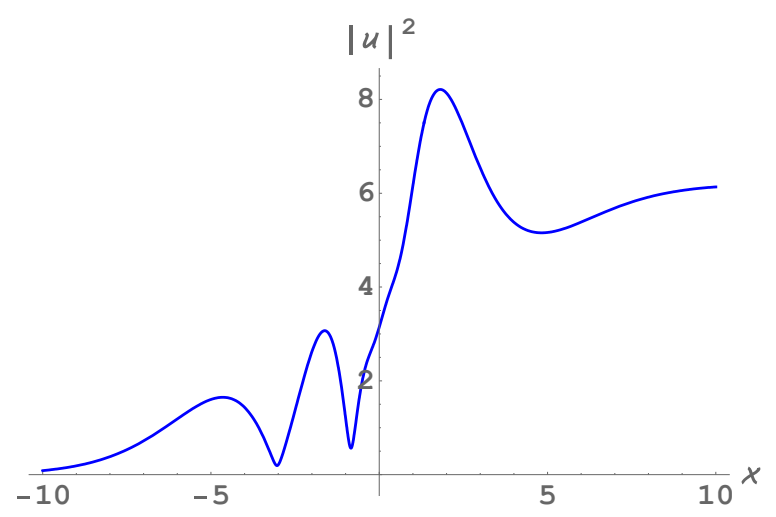

Fig. 9: The graph of probability density of spacetime- fractional SG equation with $6^{\text {th }}$ order of Taylor series.

\section{Conclusion}

In this study, the behaviours of some physical systems which have ordinarily been described by the SG equation are intended to be reinvestigated under conditions closer to the real world. These are the physical systems having memory effects of non-Markovian processes, strong interactions between particles of the systems and environment and some physical losses. This aim is tried to be realized by describing these systems with fractional SG equations. Time-, space- and time-space-fractional SG equations are all defined using Caputo derivative operator. Both the classical and fractional SG equations are solved using HPM for integer and fractional (up to $14^{\text {th }}$ terms) Taylor series of sine function, respectively and the probability densities belonging to the obtained solution functions are plotted against time and space and also the fractional parameters. For the classical SG equation, the probability density graph has naturally the form of conserved probability density. This is consistent with the fact that classical SG equation describes homogeneous systems. The classical SG equation represents the instantaneous state of the system. According to the obtained results when the numbers of terms of Taylor series increase, the difference between the obtained results steadily gets smaller. Especially, time- fractional SG equation approaches the classical SG equation and, it was observed that the $\alpha$ dependence of solution is almost eliminated. The solution moves away from the classical one for the lowest valued parameter $\alpha=1.2$ for all terms. The difference in the spacetime- fractional SG equation for same parameter in all terms is very little.

However, as is clearly observed from the solutions of fractional SG equations, the probability densities of the solution functions are nonGaussian. This shows that the systems are inhomogeneous, irreversible and they bear nonMarkovian effects. Strong interactions between the particles in the systems, the systemenvironment interaction, some physical losses such as friction and all-history of the systems give birth to such properties. Kink of type solution was obtained for both classical and time-fractional SG equations. But the results for time-fractional SG equations are different from that of classical one, depending on the fractional parameter $\alpha$. The graphical representations show that when the fractional parameter gets closer to the integer values, the results tend nearly the same with the classical SG equation. The interesting result was achieved for space-fractional SG equation; because breather of type solution was obtained unlike the others. It means a coupled kink and antikink soliton. Kink of type soliton is nonlocalized and particle of type. Consideration of space-fractional derivative operator in SG equation transforms the structure type of the solution from a non-localized particle to a localized non-linear wave. Thus losses at signal dispersion in the fiber optics may be explained with this approximation. Also when the order of Taylor series of sine function increases the result is the same as classical one. Dependence of fractional parameter disappears for timefractional SG equation.

\section{References}

Akgul A., Inc M., Karatas E. and Baleanu D., 2015. Numerical solutions of fractional differential 
equations of Lane-Emden type by an accurate technique. Advances in Difference Equations, 2015, 220.

Akgul A., Inc M., Kilicman A. and Baleanu D., 2016. A new approach for one-dimensional sine-Gordon equation. Advances in Difference Equations, 2016, 8.

Aktosun T., Demontis F. and Mee C., 2010. Exact solutions to the Sine-Gordon Equation. Journal of Mathematical Physics, 51, 123521.

Ateş I. and Yıldırım A., 2010. Applications of variational iteration and homotopy perturbation methods to obtain exact solutions for timefractional diffusion-wave equations. International Journal of Numerical Methods for Heat \& Fluid Flow, 20(6), 638.

Batiha B. et al., 2007. Numerical solution of sineGordon equation by variational iteration method. Physics Letters A, 370, 437-440.

Bracken P., 2011. Surfaces of Arbitrary Constant Negative Gaussian Curvature and Related SineGordon Equations. Mathematica Aeterna, 1 (11), 1-11.

Caputo M.,1967. Linear model of dissipation whose $Q$ is almost frequency independent-II. Geophys. J. R. Astron. Soc., 13, 529-539.

Derks G., Doelman A., Gils S., Visser T., 2003 Travelling waves in a singularly perturbed sineGordon equation. Physica D, 180, 40-70.

Elsaid A., Hammad D., 2012. Measurable Lipschitz selections and set-valued integral equations of fractional order. Journal of Fractional Calculus and Applications, 2, 1-8.

El-Shahed M., 2005. Application of He's homotopy-perturbation method to Volterra's integro-differential equation. Int. J. Nonlinear Sci. Numer. Simul., 6, 163-168.

Frenkel J., Kontorova T., 1939. On the theory of plastic deformation and twinning. Journal of Physics (USSR), 1, 137-149.

He J. H., 2004. The homotopy perturbation method for nonlinear oscillators with discontinuities. Appl. Math. Comput, 151, 287292.
He J. H., 2000. A coupling method of a homotopy technique and a perturbation technique for nonlinear problems. Internat. J. Non-Linear Mech., 35, 37-43.

He J. H., 2005. Application of homotopy perturbation method to nonlinear wave equations. Chaos Solitons Fractals, 26, 695-700.

He J. H., 2005, Homotopy perturbation method for bifurcation of nonlinear problems. Int. J. Nonlinear Sci. Numer. Simul, 6, 207-208.

He J. H., 2005. Periodic solutions and bifurcations of delay differential equations. Phys. Lett. A, 374, 228-230.

Ivancevic Vladimir G and Ivancevic Tijana T., 2013. Sine-Gordon Solitons, Kinks and Breathers as Physical Models of Nonlinear Excitations in Living Cellular Structures. Journal of Geometry and Symmetry in Physics (JGSP), 31, 1-56.

Jin L. 2009. Analytical approach to the sineGordon equation using homotopy perturbation method. Int. J. Contemp. Math. Sciences, 4 (5) 225-231.

Kaya D 2003. A numerical solution of the sineGordon equation using the modified decomposition method. Applied Mathematics and Computation, 143, 309 .

Lu J., 2009.An analytical approach to the sineGordon equation using the modified homotopy perturbation method. Computers and Mathematics with Applications, 58, 2313-2319.

Metzler R., Klafter J., 2000. The random walks guide to anomalous diffusion: A fractional dynamics approach. Phys. Rep., 339, 1-77.

Miller K. S, Ross B., An Introduction to the Fractional Calculus and Fractional Differential Equations. New York, John Wiley Sons.

Miskinis L., 2005. The Nonlinear and Nonlocal Integrable Sine-Gordon Equation. Mathematical Modelling and Analysis, 479, 483.

Momani S., Odibat Z., 2007. Homotopy perturbation method for nonlinear partial differential equations of fractional order. Phys. Lett. $A, \mathbf{3 6 5}, 345$. 
Nasrolahpour H., 2013. Fractional Lagrangian and Hamiltonian formulations in field theory Generalized multiparameters fractional variational calculus. Prespacetime Journal, 4, 604-608.

Oldham K. B, Spainer J., 1974. The Fractional Calculus. San Diego California, Academic Press.

Pandir Y.and Duzgun H. H., 2016. New exact solutions for fractional Sine-Gordon equation by using the new version of generalized F-expansion method. AIP Conference Proceedings, 1738, 290020.

Podlubny I., 1999. Fractional Differential Equations. New York, Academic Press.

Saha Ray S., 2016. A new analytical modelling for nonlocal generalized Riesz fractional sine-Gordon equation. Journal of King Saud University Science, 28(1), 48-54.

Tarasov V.E., 2005. Continuous medium model for fractal media. Phys. Lett. A, 336, 167.

Tsallis C., 1988. Possible generalization of Boltzmann-Gibbs statistics. Stat. Phys, 52, 479487.

Yousef A. M. , Rida S. Z. and Ibrahim H. R., 2016. Solutions for the fractional differential coupled sine- Gordon equation with Homotopy analysis method and the modified decomposition method. Scitech Research Organisation, 6(4), 831.

Wazwaz A. M., 2012. N-soliton solutions for the sine-Gordon equation of different dimensions. J. Appl. Math. \& Informatics, 30 (5-6), 925-934.

\section{Internet sources}

1-http://pauli.unimuenster.de/tp/fileadmin/lehre/NumMethoden/ WS0910/ScriptPDE, (10.06.2015)

2http://www.fas.org/sgp/othergov/doe/lanl/pubs/ 00285753.pdf, (26.02.2016)

3http://young.physics.ucsc.edu/250/mathematica/ sinegordon.nb.pdf, (03.05.2014)
4http://www.researchgate.net/publication/241918 156, (28.04.2016) 